\title{
Mitigating COVID-19 in a Nationally Representative UK Sample: Personal Abilities and Obligation to Obey the Law Shape Compliance with Mitigation Measures
}

\author{
Emmeke Barbara Kooistra ${ }^{\mathrm{a}}$, Chris Reinders Folmer ${ }^{\mathrm{a}}$, Malouke Esra Kuiper ${ }^{\mathrm{a}}$, Elke Olthuis ${ }^{\mathrm{a}}$, \\ Megan Brownlee ${ }^{\mathrm{a}}$, Adam Fine ${ }^{\mathrm{b}}$, \& Benjamin van Rooij ${ }^{\mathrm{a}, \mathrm{c}^{*}}$.
}

\author{
${ }^{a}$ University of Amsterdam, School of Law \\ ${ }^{\mathrm{b}}$ Arizona State University, School of Criminology and Criminal Justice \\ ${ }^{c}$ University of California, Irvine, School of Law
}

* To whom correspondence should be addressed: $\underline{\text { b.vanrooij@uva.nl }}$

\begin{abstract}
The COVID-19 pandemic has greatly influenced daily life all over the world. The present study assesses what factors influenced inhabitants of the United Kingdom to comply with lockdown and social distancing measures. It analyses data from an online survey, conducted on April 6-8, 2020, amongst a nationally representative sample of 555 participants who currently reside in the UK. The results show that compliance depended mostly on people's capacity to comply with the rules, and the normative obligation they feel to obey the law. As such, compliance was not associated with deterrence or obedience out of fear, but rather with people's practical abilities and intrinsic motivation to comply. The paper discusses policy implications for effective mitigation of the virus.
\end{abstract}

Date: May 11, 2020

Word count (without tables, references, figure captions and endnotes): 6944

Notes: This working paper has not yet been peer reviewed. The authors declare that there is no conflict of interest. 


\section{Introduction}

To slow the spread of the COVID-19 pandemic, governments rely on stay-at-home and social distancing measures. These mitigation measures, however, will only work if authorities can ensure that citizens actually stay at home and practice social distancing when asked. As such, compliance is a core question in the world's response to the COVID-19 pandemic, and plays a vital role in reducing both mortality and overburdening health care systems (Walker et al. 2020).

Compared to other European countries, the United Kingdom (UK) authorities were relatively late in adopting stay-at-home measures and, at first, expressed doubt that they were necessary. ${ }^{2}$ This makes it an interesting location to study compliance with the mitigation measures, as it may have affected how UK citizens came to comply with the measures once adopted. This paper is the second in a series of studies into compliance with the COVID-19 mitigation measures in different jurisdictions. The previous paper showed that in the United States (US), self-reported compliance depended on people's capacity to obey the rules and the opportunity they had to break them. (Van Rooij et al. 2020). Moreover, the study showed that compliance depended on intrinsic motivations, rather than deterrence or fear of the authorities. In the current paper, we discuss the factors that influenced inhabitants of the United Kingdom to comply with the lockdown and social distancing measures in their country.

The present study answers two crucial questions in relation to these mitigation measures. First, it studies to what extent people have complied with the measures. Second, it identifies which factors actually impact compliance. In addition, administering the same survey that was used in the US (Van Rooij et al. 2020) in a nationally representative sample in the UK allows us to discuss the parallels and differences between the US and UK in compliance with COVID-19 mitigation measures. 


\section{Mitigation measures in the UK}

As soon as the first case appeared in the UK on January $31^{\text {st } 3}$ a public health information campaign was launched that advised citizens to always carry tissues, use them and to bin the tissue after usage, to wash hands with soap and water, or to use sanitizer gel to kill germs. ${ }^{4}$ At first, the UK authorities expressed doubt about implementing more vigorous measures, as they thought letting the virus spread would achieve "herd immunity", and the government aimed for persuasion of the public rather than compulsion and enforcement. ${ }^{5}$ The earliest documented transmission of COVID-19 within the UK was documented on the $28^{\text {th }}$ of February. ${ }^{6}$ On March 16, Prime Minister Boris Johnson advised all citizens to avoid nonessential travel and contact with others to mitigate the outbreak of the virus. He suggested that people should avoid pubs, clubs and theatres, and work from home as much as possible. Furthermore, he advised vulnerable people such as people over the age of 70 , adults with health issues, and pregnant women to self-isolate for 12 weeks. $^{7}$ Schools, colleges, and nurseries were closed from March 20 on. $^{8}$

On March 23, Prime Minister Johnson introduced new drastic measures in the form of a police-enforced lockdown for the entire UK for at least three weeks to fight the outbreak of COVID-19. ${ }^{9}$ This means that people are only allowed to leave their house to go shopping for basic necessities such as food and medicine, one form of exercise a day, any medical need, to provide care or help to a vulnerable person, or to travel to and from work when this be cannot done from home. ${ }^{10}$ People need to keep a 2 meters distance from other people at all times if they go out, and wash their hands as soon as they get home. People are advised not to meet others outside their direct household, even friends or relatives. ${ }^{11}$ On April 16, the lockdown measure was extended for at least another three weeks. ${ }^{12}$

The UK used several approaches to ensure compliance with these measures. A first approach was to issue fines in order to deter people from violating the rules. In the case 
people do leave their home or gather in public for any other reason than those given, they risk a fine of $£ 60$, (which may be lowered to $£ 30$ if they pay within 14 days). This fine will be doubled on each further repeat offence with a maximum of $£ 960 .{ }^{13}$ To enforce the rules, apart from ordinary human patrols, police also used drones, posting footage of people breaking the rules in an attempt to deter others. ${ }^{14}$ These are principles grounded in classic deterrence theory (Nagin 2013, Becker 1968).

Authorities also focused on raising awareness and moral support for the measures. The BBC, the UK's main public TV broadcaster, for instance has created a poster that summarizes the measures in eight clear pictures with short texts, under a heading of "How can I help slow the spread of the virus?"15 The UK National Health Service (NHS), for instance, developed posters that read: "Coronavirus, Stay Home, Protect the NHS and Save Lives." ${ }^{16}$ Prime Minister Boris Johnson tweeted on April 4: "[...] but in this fine weather, please don't be tempted to break the regulations. I urge everyone to stay at home, protect the NHS and save lives." ${ }^{\prime 17}$ Authorities closed down public venues to ensure people cannot gather easily in public, and when people continued to gather in large groups in parks, some park authorities decided to close their gates as well. ${ }^{18}$ Similarly, some beaches closed parking, to prevent people to gather in larger groups to enjoy the spring weather. ${ }^{19}$ The question remains whether deterrence, moral support, or other psychological or situational factors actually promoted compliance.

\section{Present study}

The present paper analyses the results from a survey conducted in a nationally representative sample in the UK. The survey, which was also conducted earlier in the United States (Van Rooij et al. 2020), focuses on two core contagion mitigation measures. The first are social distancing measures. Here, the survey looks at whether people keep a safe distance from others (in the UK this has been defined at 2 meters), and whether they refrain from 
meeting people outside of their own household. The second are stay-at-home-type measures (referred to as lockdown measures in the UK). While there is variation in the exact details of such measures, their core substance is identical; namely, that people are to stay at home and only go out for what are deemed essential activities (which have been defined differently in different jurisdictions). ${ }^{20}$ To study compliance with these measures in the UK, the survey uses self-reported measures of compliance by asking respondents to report the frequency that they have engaged in the behaviours that are desired (or prohibited) by the measures.

In addition to examining the level of compliance with these measures, the study also explores the processes that may shape compliance. To do so, we draw from insights about compliance and why people obey or break rules from psychology, criminology, sociology, and economics (Van Rooij and Sokol 2021 (Forthcoming), Friedman 2016, Feldman 2018). This is different from previous COVID-19 compliance studies that have not reported what shapes variation in compliance (I\&O Research and Universiteit Twente 2020, Fetzer et al. 2020), or only measured behavioural intentions (Lunn et al. 2020, Everett et al. 2020). Other studies that have assessed variation in compliance with COVID-19 mitigation measures found that compliance is shaped by general personality characteristics, perceived social norms and attitudes towards the measures (Bogg and Milad 2020), political orientation (Kushner Gadarian, Wallace Goodman, and Pepinsky 2020), trust in science (Plohl and Musil 2020), and fear of the virus or attitudes about its severity (Oosterhoff and Palmer 2020, Harper et al. 2020), but not on a comprehensive operationalization of variables that shape compliance from the existing compliance literatures (Van Rooij and Sokol 2021 (Forthcoming)).

The independent variables that are included in the present study comprise substantive moral support for the measures (e.g., Tyler 1997, 2006), costs of compliance (e.g., Paternoster and Simpson 1993, Donovan and Blake 1992, Botchkovar, Tittle, and Antonaccio 
2009), deterrence (e.g., Nagin 2013, Apel 2013), capacity to comply (e.g., Van Rooij 2021 (Forthcoming)), opportunity to violate the measures (Cohen and Felson 1979, Clarke 1980), impulsivity (Gottfredson and Hirschi 1990, Pratt and Lloyd 2021 (forthcoming)), descriptive social norms (e.g., Cialdini and Goldstein 2004, Schultz et al. 2007), obligation to obey the law (e.g., Fine, Van Rooij, et al. 2016, Posch et al. 2020, Tyler 2017, Fine et al. 2020), and political orientation (e.g., Prior 2013, Spohr 2017). For a detailed reasoning behind each of these variables, see Van Rooij et al. (2020).

\section{Methods}

Ethical approval was obtained from the Institutional Review Board of the University of California, Irvine, on April 1, 2020. All participants provided consent before participating in the study. Participation was voluntary, and all participants could stop the survey at any time.

\section{Sample}

$N=579$ UK residents completed the survey in Qualtrics. Participants were recruited through the online platform Prolific Academic, using a nationally representative stratified sampling method on age, gender, and ethnicity. By definition, Prolific samples are nonprobability convenience samples. While no sample will ever be truly nationally representative (Couper 2017, Zhang et al. 2017) it is possible to create nationally representative samples on key characteristics through stratified sampling. Prolific takes the intended sample size and screens participant eligibility for representative samples using three metrics based on data from the UK Office of National Statistics: age, sex, and ethnicity. The platform stratifies age using five 9-year brackets: $18-27,28-37,38-47,48-57$, and $58+$. Sex is stratified into male and female. Ethnicity is stratified into the five categories recommended by the UK Office of National Statistics: White, Mixed, Asian, Black and Other. Participants are then screened and 
entered into the survey to fill each stratification level. While opt-in internet surveys do have non-response error (Couper et al. 2018) and internet use is not universal (Couper 2017), both of which contribute to issues of coverage and sampling, they can be as accurate as random digit dial telephone surveys (Ansolabehere and Schaffner 2014, Hines, Douglas, and Mahmood 2010) and social desirability may be lower for internet samples versus offline samples (Zhang et al. 2017).

The data was collected from April $6^{\text {th }}-8^{\text {th }} 2020$. Participants were paid $£ 2,44$ for participation. Twenty participants were excluded because they provide professional care for COVID-19 patients, and four participants were excluded because they failed the attention check. Table 1 shows the characteristics of the final sample $(N=555)$.

Table 1.

Sample characteristics for the United Kingdom.

\begin{tabular}{ll}
\hline \multicolumn{2}{l}{ Sample characteristics for the United Kingdom. } \\
\hline Age (Mean $(S D))$ & $46.22(15.57)$ \\
Gender & $51.0 \%$ \\
$\quad$ Female & $48.6 \%$ \\
Male & $0.4 \%$ \\
Other (non-binary) & \\
Ethnicity & $83.2 \%$ \\
$\quad$ Ethnic majority & $15.3 \%$ \\
Ethnic minority & \\
Education & $4.7 \%$ \\
$\quad$ No diploma & $36.6 \%$ \\
High school degree & $58.7 \%$ \\
$\quad$ College degree and higher & \\
Political orientation & $17.5 \%$ \\
$\quad$ Very progressive & $31.9 \%$ \\
$\quad$ Slightly progressive & $30.6 \%$ \\
$\quad$ Slightly conservative & $7.2 \%$ \\
$\quad$ Very conservative & \\
\hline Nb. Ethnicity and Political orientation - percentages may \\
not add up to 100\% as subjects could select the option to \\
"prefer not to say".
\end{tabular}

\section{Materials}

Control variables. The following descriptive statistics were recorded: age, gender, nationality, information on residency (country, city), employment status, occupation, education, household residents (total number and number of children), ethnicity, and Social Economic Status before and after COVID-19 (MacArthur Scale of Subjective Social Status; Adler et al. 2000). Furthermore, participants indicated whether they provide professional care 
for patients of COVID-19, whether they visited friends or family over the age of 75 on a regular basis prior to the outbreak of the virus, and whether a) they, or b) anyone they know have underlying health issues that make them more at-risk for contracting COVID-19. Finally, participants were asked to indicate their trust in science on a single item taken from McCright et al. (2013), on 5-point Likert scale from (1) "completely distrust" to (5) "completely trust", and trust in media reporting on a single item similar to the trust in science item. Table 2 and 3 show the descriptive statistics of the control and independent variables, respectively.

Compliance with COVID-19 measures. Compliance was measured on two main COVID-19 mitigation measures: "social distancing" and "lockdown" measures. On all items, participants answered on a 7-point Likert scale ranging from (1) "never" to (7) "always". Four items $(\alpha=.52)$ measured whether participants complied with social distancing measures. Compliance with lockdown measures was measured with a single item. Initially, mean scores were calculated both for the 4-item social distancing scale and the single-item lockdown measure together (5-items; $\alpha=.62)$.

Because our dependent variable, the combined compliance measure, had weak internal consistency $(\alpha=.62)$, we ran a factor analysis and found that two items for social distancing loaded on a separate factor. Yet even after removing these items, the Cronbach's alpha for the remaining three items did not increase $(\alpha=.62)$. As the items did not produce one measure for compliance, we decided to analyse the questions separately. Two items were left out of these analysis as their content (i.e., "I still visit others (friends or relatives) to outside of my direct household.", "I still allow others (friends or relatives) to visit my direct household.") was completely covered by one of the other items ("I still meet people outside of my direct household."). Therefore, it was decided to only include the last item, whether people refrained from "meeting people". Thus, we focused the analyses on three single 
questions that assess separate aspects of compliance with the mitigation measures: 1) Meeting with people from outside one's household ("I still meet people outside of my direct household." (reverse scored)); 2) Keeping a safe distance from people ("I keep a safe distance from people outside of my direct household."); and 3) Staying home under the lockdown orders ("I have stayed at home after I was ordered to do so, apart from engaging in essential activities (e.g., grocery shopping, medical appointments).”). Higher values indicate greater compliance with each of the three COVID-19 mitigation measures. Table 4 shows the descriptive statistics of the compliance with COVID-19 measures. For all items means are considerably large, indicating that overall, participants self-reported high rates of compliance.

Substantive moral support. Substantive moral support was measured on three subscales; perceived threat, specific moral alignment, and support for current policies.

Perceived threat. Perceived threat was measured using three items $(\alpha=.89)$ on which the participants indicated to what extent they believed the Coronavirus was a threat to themselves or others, rated on a 7-point Likert scale ranging from (1) "very strongly disagree" to (7) "very strongly agree".

Specific moral alignment. Specific moral alignment was measured using two items ( $\alpha$ $=.96)$ on which participants indicated to what extent they morally believed people should follow the COVID-19 mitigation measures, rated on a 7-point Likert scale ranging from (1) "very strongly disagree" to (7) "very strongly agree".

Support for current policies. Support for current policies was measured using three items $(\alpha=.28)$ on which participants indicated to what extent they supported the authorities in adopting the COVID-19 mitigation measures, rated on a 7-point Likert scale ranging from (1) "very strongly disagree" to (7) "very strongly agree". One item was reverse coded. The mean score was calculated and higher values indicate more support for the authorities 
adopting the COVID-19 measures. One item correlated poorly. If removed, Cronbach's alpha rose to $\alpha=.85$, and this item was excluded from further analysis.

Costs of compliance and strain. Costs of compliance and strain were measured on two sub-scales: costs of compliance and negative emotions.

Costs of compliance. Participants indicated on five items $(\alpha=.78)$ how likely it was that compliance with the COVID-19 mitigation measures would have a negative financial and social impact on them. Participants answered on a 7-point Likert scale ranging from (1) "extremely unlikely" to (7) "extremely likely".

Negative emotions. Negative emotions due to COVID-19 was measured on six items $(\alpha=.88)$ assessing different negative emotions, rated on a 7-point Likert scale ranging from (1) "very strongly disagree" to (7) "very strongly agree". Means were calculated and higher values indicate higher negative emotions.

Deterrence. Deterrence was measured for the two COVID-19 mitigation measures separately. For each, two items (social distancing $\alpha=.71$, lockdown $\alpha=.84$ ) measured certainty of apprehension and punishment for violating COVID-19 measures, answered on a 7-point Likert scale ranging from (1) "extremely improbable" to (7) "extremely probable". Furthermore, for each mitigation measure, participants indicated on a single item how much suffering punishment for violating COVID-19 measures would cause them, in order to measure the subjective severity of punishment, answered on a 6-point Likert scale ranging from (1) "extreme suffering" to (6) "no suffering at all". Lower scores indicate higher suffering. We observed strong associations between deterrence perceptions for social distancing and lockdown measures. As such, these responses were combined into aggregated scale measures of punishment certainty $(\alpha=.87)$ and punishment severity $(\alpha=.88)$. 
Capacity to comply. The capacity to comply was measured using three sub-scales: the practical capacity to comply, the perceived clarity of the current measures, and knowledge of the current measures.

Practical capacity to comply. Three items $(\alpha=.60)$ measured to what extent participants are practically able to comply with the COVID-19 mitigation measures, on a 7point Likert scale ranging from (1) "very strongly disagree" to (7) "very strongly agree". One item correlated poorly $(\alpha=.68$ if deleted). This item was excluded from further analysis.

Perceived clarity of current measures. Furthermore, participants were asked whether the measures adopted by the authorities to reduce the spread of COVID-19 were clear to them, answered on a 7-point Likert scale ranging from (1) "extremely unclear" to (7) "extremely clear", where higher scores indicate greater clarity

Knowledge of current measures. Participants indicated for seven statements which COVID-19 mitigation measures currently apply to them, with three answer options (yes/no/unsure). As all measures were in force within the UK when the survey was conducted, the total number of "yes" answers given (range 0-7) was used as an indicator of legal knowledge.

Opportunity to violate. Five items $(\alpha=.82)$ measured to what extent participants in practice had the opportunity to violate the COVID-19 mitigation measures, on a 7-point Likert scale ranging from (1) "very strongly disagree" to (7) "very strongly agree".

Impulsivity. Impulsivity was measured using a subset of five items $(\alpha=.76)$ taken from the 8-item impulse control subscale from the Weinberger Adjustment Inventory (WAI; Weinberger and Schwartz 1990, Fine, Steinberg, et al. 2016). The items are answered on a 5point Likert scale ranging from (1) "false" to (5) "true". One item was reverse coded and means were calculated such that higher scores indicate higher impulsivity. 
Descriptive Social norms. Participants rated to what extent people they know comply with the COVID-19 measures (one item for each measure, $\alpha=.94$ ), on a 7-point Likert scale ranging from (1) "very strongly disagree" to (7) "very strongly agree". Higher scores indicate more compliant descriptive social norms.

Obligation to obey the law (OOL). To assess OOL the survey included three subscales: Normative obligation to obey the law, non-normative obligation to obey, and procedural justice.

Normative obligation to obey. Normative obligation to obey was measured with a single item, "I feel like it is sometimes okay to break the law," on a 7-point Likert scale ranging from (1) "strongly agree" to (7) "strongly disagree". This item was created for the current study based on extant work (e.g., Estévez and Emler 2010, Reisig, Bratton, and Gertz 2007, Fine et al. 2020). Higher scores indicate greater normative obligation to obey the law.

Non-normative obligation to obey. Non-normative obligation was measured using a single item: "I only obey the authorities handling the Coronavirus because I am afraid of them." (adapted for COVID-19 following Posch et al. 2020, Tankebe, Reisig, and Wang 2016), measured on a 7-point Likert scale ranging from (1) "very strongly disagree" to (7) "very strongly agree". Higher scores indicate higher non-normative obligation to obey.

Procedural justice (PJ). Procedural justice was measured by using items adapted from prior measures of procedural justice used in the study of police (Baker and Gau 2018, Gau 2014, Tyler 1997, Wolfe et al. 2016). Three items $(\alpha=.93)$ measured PJ in creating the COVID-19 mitigation measures. Four items $(\alpha=.93)$ measured PJ in enforcing the COVID19 mitigation measures. The items were answered on a 7-point Likert scale ranging from (1) "very strongly disagree" to (7) "very strongly agree". Because PJ perceptions for creation and enforcement were strongly correlated, we calculated means for a combined scale $(\alpha=.95)$, with higher values indicating higher procedural justice. 
Political orientation. Political orientation was measured with a single item adapted from Hasson et al. (2018), Fine, Rowan, and Simmons (2019) and Wojcik et al. (2015). There were four answer possibilities, ranging from (1) "very progressive" to (4) "very conservative". Additionally, participants could indicate that they "prefer not to say"; these were excluded from analyses that included political orientation. ${ }^{21}$

\section{Results}

\section{Descriptive Statistics and Correlations}

Table 2 and 3 show the descriptive statistics of the control and independent variables, respectively. Table 4 provides descriptive statistics on the dependent variables. Table 5 shows the correlations between the compliance measures and the control variables. Table 6 shows the correlations between the compliance measures and the independent variables.

Table 2.

Descriptive statistics of the control variables $(N=555)$.

\begin{tabular}{lll}
\hline & Mean $(S D)$ & Scale \\
\hline$N$ Household & $2.88(1.42)$ & \\
$N$ Children & $.57(.98)$ & \\
& & \\
SES pre-COVID-19 & $5.29(1.63)$ & $1-10$ \\
SES post-COVID-19 & $5.11(1.75)$ & $1-10$ \\
& & \\
Friends/Family 75+ & $46.7 \%$ & \\
Health issues self & $30.1 \%$ & \\
Health issues other & $80.9 \%$ & \\
& & \\
Trust in science & $4.15(.90)$ & $1-5$ \\
Trust in media & $2.72(1.17)$ & $1-5$ \\
\hline
\end{tabular}

Table 3.

Descriptive statistics of the independent variables $(N=555)$.

\begin{tabular}{lccc}
\hline & Mean & SD & Scale \\
\hline Substantive moral alignment & & & \\
$\quad$ Perceived threat & 5.71 & 1.21 & $1-7$ \\
$\quad$ Specific moral alignment & 6.54 & 1.04 & $1-7$ \\
$\quad$ Support for current policies & 4.35 & 1.34 & $1-7$ \\
Costs of compliance & 4.17 & 1.40 & $1-7$ \\
$\quad$ Negative emotions & 4.45 & 1.15 & $1-7$ \\
Deterrence & & & \\
$\quad$ Certainty & 3.88 & 1.32 & $1-7$ \\
$\quad$ Severity & 3.83 & 1.20 & $1-6$ \\
Capacity to comply & 5.79 & 1.32 & $1-7$ \\
$\quad$ Clarity current measures & 5.57 & 1.42 & $1-7$ \\
$\quad$ Knowledge current measures & 6.78 & .63 & $0-7$ \\
Opportunity to violate & 3.50 & 1.42 & $1-7$ \\
Impulsivity & 1.93 & .71 & $1-5$ \\
Social Norms & 5.77 & 1.21 & $1-7$ \\
Obligation to obey the law & & & \\
$\quad$ Normative OOL & 5.04 & 1.74 & $1-7$ \\
$\quad$ Non-normative OOL & 2.28 & 1.28 & $1-7$ \\
$\quad$ Procedural Justice & 5.09 & 1.19 & $1-7$ \\
Political Orientation & 2.32 & .89 & $1-4$ \\
\hline Nb. Political Oriention
\end{tabular}

Nb. Political Orientation $-N=484$.

Table 4.

Descriptive statistics of Compliance with COVID-19 measures (Likert 1-7, never - always) $(N=555)$.

\begin{tabular}{llrr}
\hline Item & Mean & SD \\
\hline Since the authorities took measures to contain the Coronavirus: & & \\
Meeting people: & "I still meet people outside of my direct household."^ & 6.47 & 1.08 \\
Safe distance: & "I keep a safe distance from people outside of my direct household." & 6.41 & 1.09 \\
Lockdown: & "I have stayed at home after I was ordered to do so, apart from engaging & 6.51 & 1.13 \\
\hline & in essential activities (e.g., grocery shopping, medical appointments)." & & \\
\hline
\end{tabular}

Nb. ${ }^{\wedge}-$ Reverse scored. 
Table 5.

Kendall's tau correlations with control variables $(N=555)$.

\begin{tabular}{|c|c|c|c|c|c|c|c|c|c|c|c|c|}
\hline & 总 & 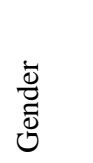 & 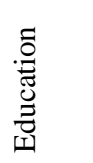 & 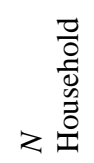 & $\begin{array}{l}\bar{D} \\
\frac{0}{\Xi} \\
\bar{\Xi} \\
z\end{array}$ & 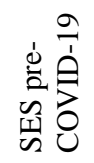 & 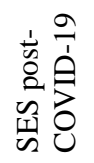 & 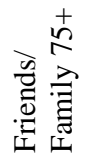 & 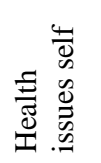 & 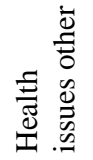 & 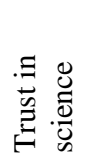 & 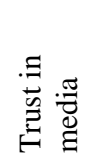 \\
\hline Gender & .030 & & & & & & & & & & & \\
\hline Education & .004 & -.004 & & & & & & & & & & \\
\hline$N$ Household & $-.339^{* *}$ & .018 & -.063 & & & & & & & & & \\
\hline$N$ Children & $-.129^{* *}$ & -.001 & .028 & $.525^{* *}$ & & & & & & & & \\
\hline SES pre-COVID-19 & .005 & .043 & $.168^{* *}$ & .030 & .017 & & & & & & & \\
\hline SES post-COVID-19 & .008 & .000 & $.131^{* *}$ & .022 & -.001 & $.794^{* * *}$ & & & & & & \\
\hline Friends/Family 75+ & $.094^{* *}$ & .072 & .073 & -.023 & -.006 & $.118^{* * *}$ & $.093^{*}$ & & & & & \\
\hline Health issues self & $.181^{* *}$ & .022 & -.054 & $-.090^{*}$ & -.011 & $-.092^{*}$ & $-.092^{*}$ & .032 & & & & \\
\hline Health issues other & .012 & $.092^{*}$ & .044 & -.020 & -.051 & .032 & .038 & $.188^{* *}$ & $.159^{* *}$ & & & \\
\hline Trust in science & -.014 & -.048 & .049 & -.029 & -.037 & $.113^{* *}$ & $.113^{* *}$ & -.004 & .043 & $.089^{*}$ & & \\
\hline Trust in media & $.083^{* *}$ & .047 & .016 & -.037 & .052 & .061 & .035 & .006 & -.033 & -.071 & $.207^{* *}$ & \\
\hline Compliance & & & & & & & & & & & & \\
\hline Meeting people & -.034 & $.095^{*}$ & -.062 & .028 & -.027 & .012 & .000 & -.025 & .067 & .060 & -.004 & .016 \\
\hline Safe distance & $.121^{* *}$ & $.130^{* *}$ & .006 & -.009 & -.002 & .025 & -.011 & .023 & $.112^{* *}$ & $.110^{* *}$ & .069 & $.075^{*}$ \\
\hline Lockdown & .023 & $.189^{* *}$ & -.031 & -.022 & -.008 & $.073^{*}$ & .032 & -.030 & $.119^{* *}$ & .067 & .045 & .057 \\
\hline
\end{tabular}


Table 6.

Kendall's tau correlations with the independent variables $(N=555)$.

\begin{tabular}{|c|c|c|c|c|c|c|c|c|c|c|c|c|c|c|c|c|c|}
\hline & 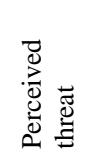 & 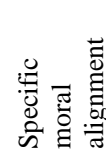 & 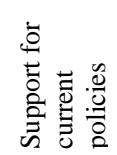 & 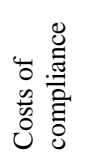 & 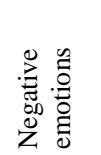 & 量 & 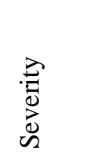 & 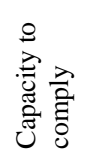 & 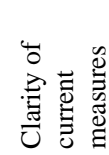 & 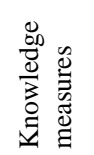 & 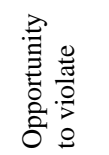 & 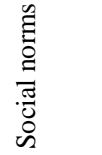 & 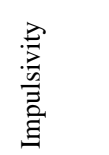 & 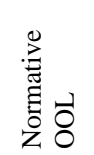 & 突 & 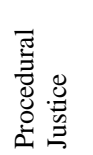 & 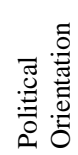 \\
\hline Specific moral alignment & $.395^{* *}$ & & & & & & & & & & & & & & & & \\
\hline Support for current policies & $.119^{* * *}$ & $.074^{*}$ & & & & & & & & & & & & & & & \\
\hline Costs of compliance & -.018 & -.029 & -.050 & & & & & & & & & & & & & & \\
\hline Negative emotions & $.220^{* * *}$ & $.089^{* *}$ & -.008 & $.162^{* *}$ & & & & & & & & & & & & & \\
\hline $\begin{array}{l}\text { Deterrence } \\
\quad \text { Certainty }\end{array}$ & $.097^{* * *}$ & .022 & $.155^{* *}$ & .058 & $.061^{*}$ & & & & & & & & & & & & \\
\hline Severity & $-.084^{* * *}$ & .005 & -.041 & $-.080^{*}$ & $-.117^{* *}$ & $-.203^{* *}$ & & & & & & & & & & & \\
\hline Capacity to comply & $.166^{* *}$ & $.285^{5^{* *}}$ & .051 & -.038 & .008 & .054 & -.008 & & & & & & & & & & \\
\hline Clarity current measures & $.144^{* *}$ & $.232^{2 *}$ & $.365^{* * *}$ & $-.081^{*}$ & -.056 & $.134^{* *}$ & .003 & $.195^{* *}$ & & & & & & & & & \\
\hline Knowledge current measures & $.073^{*}$ & $.218^{* *}$ & .057 & -.050 & .012 & $.069^{*}$ & -.018 & $.177^{* *}$ & $.153^{* *}$ & & & & & & & & \\
\hline Opportunity to violate & $-.073^{*}$ & $-.077^{*}$ & $-.133^{* *}$ & -.011 & $-.081^{* *}$ & $-.159^{* *}$ & $.119^{* *}$ & $-.135^{* *}$ & $-.159^{* * *}$ & -.059 & & & & & & & \\
\hline Social norms & $.171^{* *}$ & $.164^{* *}$ & $.127^{* *}$ & -.051 & .025 & .060 & -.041 & $.250^{* *}$ & $.217^{* *}$ & .072 & $-.120^{* * *}$ & & & & & & \\
\hline $\begin{array}{l}\text { Impulsivity } \\
\text { Obligation to obey the law } \\
\quad \text { Normative OOL }\end{array}$ & $\begin{array}{l}-.077^{*} \\
.232^{* * *}\end{array}$ & $\begin{array}{l}-.108^{* *} \\
.211^{* *}\end{array}$ & $\begin{array}{l}-.008 \\
.208^{* * *}\end{array}$ & $\begin{array}{c}.026 \\
-.073^{*}\end{array}$ & $\begin{array}{l}.059^{*} \\
.077^{*}\end{array}$ & $\begin{array}{l}-.036 \\
.184^{* *}\end{array}$ & $\begin{array}{l}-.028 \\
-.067^{*}\end{array}$ & $\begin{array}{l}-.052 \\
.117^{* *}\end{array}$ & $\begin{array}{l}-.090^{* *} \\
.275^{* *}\end{array}$ & $\begin{array}{l}.021 \\
.058\end{array}$ & $\begin{array}{c}.020 \\
-.154^{* *}\end{array}$ & $\begin{array}{l}-.088^{* *} \\
.159^{* *}\end{array}$ & $-.189^{* * *}$ & & & & \\
\hline Non-normative $O O L$ & -.046 & $-.214^{* *}$ & .025 & $.094^{* *}$ & .059 & $.105^{* *}$ & $-.075^{*}$ & $-.179^{* *}$ & $-.105^{4 *}$ & $-.087^{*}$ & -.026 & $-.144^{* * *}$ & $.098^{* * *}$ & -.017 & & & \\
\hline Procedural Justice & $.138^{* *}$ & $.176^{4 *}$ & $.322^{* * *}$ & $-.117^{* *}$ & .009 & $.130^{* *}$ & .009 & $.191^{* *}$ & $.327^{* * 4}$ & $.095^{* *}$ & $-.117^{* * *}$ & $.220^{* * *}$ & $-.104^{* * *}$ & $.264^{* *}$ & $-.108^{* *}$ & & \\
\hline Political orientation & .033 & -.051 & $.319^{* *}$ & -.063 & -.060 & .042 & -.009 & -.033 & $.221^{* *}$ & .002 & -.035 & .057 & -.040 & $.187^{* *}$ & $.082^{*}$ & $.216^{* *}$ & \\
\hline $\begin{array}{l}\text { Compliance } \\
\text { Meeting people }\end{array}$ & $.107^{* *}$ & $.293^{* *}$ & .051 & -.016 & .064 & .024 & .043 & $.231^{* *}$ & $.120^{* *}$ & $.132^{* * *}$ & $-.099^{* * *}$ & $.107^{* * *}$ & -.009 & $.131^{* *}$ & -.056 & $.087^{*}$ & -.062 \\
\hline Safe distance & $.165^{* *}$ & $.237^{7 *}$ & .067 & .025 & .040 & .061 & -.042 & $.261^{* *}$ & $.124^{* *}$ & $.103^{* *}$ & $-.127^{* * *}$ & $.111^{* *}$ & -.045 & $.151^{* *}$ & -.060 & $.099^{* * *}$ & -.038 \\
\hline Lockdown & $.117^{* *}$ & $.313^{* *}$ & $.070^{*}$ & -.027 & .006 & .032 & -.007 & $.232^{* * *}$ & $.155^{* *}$ & $.225^{* *}$ & $-.092^{* * *}$ & .063 & $-.077^{*}$ & $.205^{* *}$ & $-.123^{* *}$ & . $092^{* * *}$ & .012 \\
\hline
\end{tabular}




\section{Regression analysis}

We performed a series of ordinary least-squares regressions using the three compliance measures as dependent variables. The predictors were each included separately in regression models, and each controlled for the same set of control variables that had significantly correlated with at least one of the compliance measures (see Table 5; age, gender, SES pre-COVID-19, health issues self, health issues other and trust in media). Regressions were adjusted for heteroscedasticity using Huber/White robust standard error estimation. Table 7 shows the results of the OLS regressions.

Table 7.

Separate OLS regression analyses of compliance $(N=555)$, adjusted for control variables

\begin{tabular}{|c|c|c|c|c|c|c|c|c|c|}
\hline & \multicolumn{3}{|c|}{ Meeting people } & \multicolumn{3}{|c|}{ Safe distance } & \multicolumn{3}{|c|}{ Lockdown } \\
\hline & B & SE & RSq & B & SE & $\mathbf{R S q}$ & B & SE & $\mathbf{R S q}$ \\
\hline \multicolumn{10}{|l|}{ Substantive moral support } \\
\hline Perceived threat & $.09 *$ & .04 & .02 & $.08 *$ & .04 & .03 & .02 & .05 & .04 \\
\hline Specific moral alignment & $.16^{* *}$ & .05 & .03 & $.15^{*}$ & .06 & .04 & $.18 * *$ & .06 & .06 \\
\hline Support for current policies & .01 & .04 & .01 & .05 & .04 & .02 & .00 & .04 & .04 \\
\hline Costs of compliance & .03 & .03 & .01 & .03 & .04 & .02 & .05 & .04 & .04 \\
\hline Negative emotions & .03 & .04 & .01 & .02 & .04 & .02 & -.09 & .05 & .04 \\
\hline \multicolumn{10}{|l|}{ Deterrence } \\
\hline Certainty & .02 & .04 & .01 & .04 & .04 & .02 & .02 & .05 & .04 \\
\hline Severity & .04 & .04 & .01 & -.02 & .04 & .02 & -.02 & .04 & .04 \\
\hline Capacity to comply & $.19 * * *$ & .05 & .07 & $.14 * * *$ & .04 & .05 & $.18 * * *$ & .05 & .08 \\
\hline Clarity current measures & $.09 *$ & .04 & .03 & $.09 *$ & .04 & .03 & $.07 *$ & .04 & .04 \\
\hline Knowledge current measures & $.28 * * *$ & .08 & .04 & $.17 *$ & .07 & .03 & $.28 * * *$ & .08 & .06 \\
\hline Opportunity to violate & $-.10 * *$ & .03 & .03 & $-.11 * *$ & .04 & .04 & -.04 & .03 & .04 \\
\hline Impulsivity & -.05 & .07 & .01 & .04 & .07 & .02 & -.07 & .08 & .04 \\
\hline Descriptive social norms & .04 & .04 & .01 & .02 & .03 & .02 & -.01 & .04 & .04 \\
\hline \multicolumn{10}{|l|}{ Obligation to obey the law } \\
\hline Normative $O O L$ & $.09 * *$ & .03 & .03 & .05 & .03 & .03 & $.09 * *$ & .03 & .05 \\
\hline Non-Normative OOL & .01 & .03 & .01 & .02 & .03 & .02 & -.04 & .04 & .04 \\
\hline Procedural Justice & .08 & .05 & .02 & .08 & .05 & .03 & .00 & .05 & .04 \\
\hline Political orientation & -.08 & .06 & .02 & $-.14 *$ & .06 & .04 & -.03 & .06 & .04 \\
\hline
\end{tabular}

The data show that several independent variables significantly predicted the compliance measures (see Table 7). We found positive, significant associations with all compliance measures for specific moral alignment, practical capacity to comply, and clarity 
and knowledge of current measures. Perceived threat was positively associated with the "meeting people" and "safe distance", but not the "lockdown" measure. Normative obligation to obey the law, however, significantly predicted the "meeting people" and "lockdown" measure, but not the "safe distance" measure. We found negative, significant associations with the "meeting people" and "safe distance" measures for opportunity, while political orientation only significantly, and negatively predicted the "safe distance" measure. For all other independent variables, no significant associations with compliance were observed.

To complement these separate regression analyses, we ran a series of stepwise regressions in which multiple predictors were included in the same model. This allowed us to examine whether the predictive utility of certain independent variables remained after accounting for other variables. For these models, collinearity statistics indicated no issues with multicollinearity (all VIFs $\leq 1.84$; all tolerances $\geq .54$ ).

In the first step (model 0), we added only the control variables that significantly correlated with compliance (see Tables 8-10). In model 1, we added the measures of substantive moral support (perceived threat, specific moral alignment, and support for current policies), costs of compliance (costs and negative emotions) and impulsivity. In model 2, we added the measures of deterrence (certainty and severity) and descriptive social norms. In model 3, we entered the measures of capacity to comply (capacity and clarity of measures) and opportunity to violate. In model 4, we entered the measures of obligation to obey the law (normative OOL, non-normative OOL, and PJ). Lastly, we entered political orientation into model 5.

In all models, a significant and large positive association can be found for the nonbinary gender category. This is most likely caused by the robust standard error estimation. As the non-binary category only contained a small portion of the sample $(N=2)$, the robust method underestimated the standard error, resulting in inflated Type I error rates (false 
positives). For completion, we checked the models after excluding the non-binary category from analyses, but found only minimal differences in our models, and no differences in significance of the predictors. We therefore decided to keep the non-binary category in our models.

For compliance with the "meeting people" measure (Table 8), results for model 5 indicate that capacity, opportunity, and normative OOL were associated with compliance. This means that people who have more practical capacity to comply, have less opportunity to violate the rules, and feel greater normative obligation to obey the law comply more with the measures that tell them not to meet people outside of their own household. The final model explained $14 \%$ of variance in compliance with this measure. All other variables were unrelated to compliance in the final model.

For compliance with the "safe distance" measure (Table 9), results for model 5 indicate that age, capacity, normative OOL, and political orientation were associated with compliance. This indicates that people who are older, have more capacity to comply, feel greater normative obligation to obey the law, and have a more progressive political orientation comply more with the measures that tell them to keep a safe distance from other people. The final model explained $14 \%$ of variance in compliance with this measure. All other variables were unrelated to compliance in the final model.

For compliance with the "lockdown" measure (Table 10), results for model 5 indicate that gender, health (self), perceived threat, specific moral alignment, negative emotions, capacity, knowledge of measures, and normative OOL were associated with compliance with lockdown measures. This indicates that females (versus males), participants with underlying health issues that make them more at risk for contracting COVID-19 (versus those who do not), participants who more strongly believed people should follow the COVID-19 mitigation measures, who had more capacity to comply and better knowledge of the current measures, 
and participants who feel greater normative obligation to obey the law comply more with measures that tell them to stay at home and refrain from nonessential outings. Conversely, participants who perceive a greater threat from the virus and experience more negative emotions show less compliance with lockdown measures. The final model explained $17 \%$ of variance. All other variables were unrelated to compliance in the final model.

Lastly, we note that none of our models explain more than $17 \%$ of variance in compliance. However, predicting single dependent variables in a model is more difficult than predicting a scale or combined variable. Accordingly, when we ran these analyses on a combined compliance measure, $\mathrm{R}^{2}$ rose to $23 \%$ percent in the final model. Moreover, results were broadly consistent with our findings in the separate models, with significant associations with health (self), specific moral alignment, capacity to comply, opportunity to violate, knowledge of current measures, normative obligation to obey the law, and political orientation. 
Table 8.

Step-wise regression models of compliance "Meeting people" $(N=555)$

\begin{tabular}{|c|c|c|c|c|c|c|}
\hline & $\begin{array}{l}\text { Model } 0 . \\
\\
\text { Baseline } \\
\text { model } \\
\text { (controls } \\
\text { only) } \\
\end{array}$ & $\begin{array}{c}\text { Model } 1 . \\
+ \\
\text { Substantive } \\
\text { moral } \\
\text { support, } \\
\text { Costs of } \\
\text { compliance, } \\
\text { Impulsivity }\end{array}$ & $\begin{array}{l}\text { + Deterrence, } \\
\text { Social Norms }\end{array}$ & $\begin{array}{l}\text { + Capacity, } \\
\text { opportunity }\end{array}$ & $\begin{array}{c}\text { + Obligation to } \\
\text { obey the law }\end{array}$ & $\begin{array}{l}\text { + Political } \\
\text { orientation }\end{array}$ \\
\hline & $\mathrm{b}(\mathrm{SE})$ & $\mathrm{b}(\mathrm{SE})$ & $\mathrm{b}(\mathrm{SE})$ & $\mathrm{b}(\mathrm{SE})$ & $\mathrm{b}(\mathrm{SE})$ & $\mathrm{b}(\mathrm{SE})$ \\
\hline Age & $-.00(.00)$ & $-.00(.00)$ & $-.00(.00)$ & $-.00(.00)$ & $-.00(.00)$ & $-.00(.00)$ \\
\hline \multicolumn{7}{|l|}{ Gender } \\
\hline i. female & $.05(.09)$ & $-.01(.09)$ & $-.01(.09)$ & $-.11(.10)$ & $-.16(.10)$ & $-.16(.11)$ \\
\hline ii. non-binary & $\begin{array}{l}.50 * * * \\
(.12)\end{array}$ & $.37 * *(.14)$ & $.33^{*}(.14)$ & $.33(.18)$ & $.34 *(.15)$ & $.39 *(.16)$ \\
\hline SES pre-COVID-19 & $.00(.02)$ & $-.00(.02)$ & $-.00(.02)$ & $-.01(.02)$ & $-.01(.02)$ & $-.01(.03)$ \\
\hline Health (self) & $.14(.10)$ & $.13(.10)$ & $.12(.10)$ & $.09(.10)$ & $.08(.10)$ & $.08(.10)$ \\
\hline Health (other) & $.15(.14)$ & $.12(.14)$ & $.11(.14)$ & $.11(.13)$ & $.12(.13)$ & $.15(.15)$ \\
\hline Trust in media & $.05(.05)$ & $.03(.05)$ & $.02(.05)$ & $.01(.05)$ & $-.00(.05)$ & $-.02(.05)$ \\
\hline Perceived threat & & $.01(.04)$ & $.01(.05)$ & $.02(.04)$ & $.00(.04)$ & $-.02(.05)$ \\
\hline Specific moral alignment & & $.15 * *(.06)$ & $.15^{* *}(.06)$ & $.08(.06)$ & $.08(.06)$ & $.07(.06)$ \\
\hline $\begin{array}{l}\text { Support for current } \\
\text { policies }\end{array}$ & & $.00(.04)$ & $-.00(.04)$ & $-.04(.04)$ & $-.06(.04)$ & $-.06(.05)$ \\
\hline Cost of compliance & & $.03(.04)$ & $.03(.04)$ & $.03(.04)$ & $.04(.04)$ & $.05(.04)$ \\
\hline Negative emotions & & $.02(.05)$ & $.03(.05)$ & $.04(.05)$ & $.03(.05)$ & $.01(.05)$ \\
\hline Impulsivity & & $-.03(.07)$ & $-.02(.07)$ & $-.01(.07)$ & $.02(.07)$ & $.03(.08)$ \\
\hline $\begin{array}{l}\text { Deterrence - } \\
\text { Certainty }\end{array}$ & & & $.03(.04)$ & $-.01(.04)$ & $-.03(.04)$ & $-.02(.05)$ \\
\hline Severity & & & $.06(.04)$ & $.06(.04)$ & $.07(.04)$ & $.09(.04)$ \\
\hline Social norms & & & $.03(.03)$ & $-.02(.03)$ & $-.03(.03)$ & $-.02(.04)$ \\
\hline Capacity to comply & & & & $.15 * *(.05)$ & $.16^{* *}(.05)$ & $.19 * * *(.06)$ \\
\hline Clarity of measures & & & & $.06(.04)$ & $.05(.04)$ & $.05(.05)$ \\
\hline Knowledge & & & & $.19 *(.08)$ & $.20 *(.08)$ & $.19(.10)$ \\
\hline Opportunity to violate & & & & $-.08 *(.03)$ & $-.08 *(.03)$ & $-.08 *(.04)$ \\
\hline $\begin{array}{l}\text { Normative obligation to } \\
\text { obey the law }\end{array}$ & & & & & $.08 *(.03)$ & $.08 *(.03)$ \\
\hline Non-normative OOL & & & & & $.05(.03)$ & $.06(.03)$ \\
\hline Procedural justice & & & & & $.01(.05)$ & $.02(.06)$ \\
\hline Political orientation & & & & & & $-.09(.06)$ \\
\hline $\mathbf{R S q}$ & .01 & .04 & .04 & .11 & .13 & .14 \\
\hline$R S q$ (change) & .01 & .03 & .00 & .07 & .02 & .01 \\
\hline
\end{tabular}

Nb. * - Significant at the .05 level. $* *-$ Significant at the .01 level. $* * *$ - Significant at the .001 level. Model $5-N=484$. 
Table 9.

Step-wise regression models of compliance "Safe distance" $(N=555)$

\begin{tabular}{|c|c|c|c|c|c|c|}
\hline & $\begin{array}{l}\text { Model } 0 . \\
\\
\text { Baseline } \\
\text { model } \\
\text { (controls } \\
\text { only) }\end{array}$ & $\begin{array}{c}\text { Model } 1 . \\
+ \\
\text { Substantive } \\
\text { moral } \\
\text { support, } \\
\text { Costs of } \\
\text { compliance, } \\
\text { Impulsivity }\end{array}$ & $\begin{array}{l}\text { + Deterrence, } \\
\text { Social Norms }\end{array}$ & $\begin{array}{l}\text { + Capacity, } \\
\text { opportunity }\end{array}$ & $\begin{array}{l}+ \text { Obligation to } \\
\text { obey the law }\end{array}$ & $\begin{array}{l}+ \text { Political } \\
\text { orientation }\end{array}$ \\
\hline & $\mathrm{b}(\mathrm{SE})$ & $\mathrm{b}(\mathrm{SE})$ & $\mathrm{b}(\mathrm{SE})$ & $\mathrm{b}(\mathrm{SE})$ & $\mathrm{b}(\mathrm{SE})$ & $\mathrm{b}(\mathrm{SE})$ \\
\hline Age & $.00(.00)$ & $.00(.00)$ & $.00(.00)$ & $.00(.00)$ & $.00(.00)$ & $.01 *(.00)$ \\
\hline \multicolumn{7}{|l|}{ Gender } \\
\hline i. female & $.20 *(.09)$ & $.15(.09)$ & $.14(.09)$ & $.07(.10)$ & $.05(.10)$ & $.01(.10)$ \\
\hline ii. non-binary & $-.25(.13)$ & $-.35^{*}(.15)$ & $-.37 *(.16)$ & $-.34(.19)$ & $-.30(.17)$ & $-.26(.17)$ \\
\hline SES pre-COVID-19 & $.02(.03)$ & $.02(.03)$ & $.02(.03)$ & $.02(.03)$ & $.02(.03)$ & $.03(.03)$ \\
\hline Health (self) & $.12(.10)$ & $.09(.10)$ & $.09(.10)$ & $.07(.11)$ & $.07(.10)$ & $.11(.11)$ \\
\hline Health (other) & $.18(.12)$ & $.15(.12)$ & $.15(.12)$ & $.15(.11)$ & $.16(.11)$ & $.23(.13)$ \\
\hline Trust in media & $.02(.05)$ & $-.01(.05)$ & $-.02(.05)$ & $-.03(.05)$ & $-.04(.05)$ & $-.08(.05)$ \\
\hline Perceived threat & & $.01(.05)$ & $-.00(.05)$ & $.00(.04)$ & $-.00(.04)$ & $-.02(.05)$ \\
\hline $\begin{array}{l}\text { Specific moral } \\
\text { alignment }\end{array}$ & & $.15^{*}(.07)$ & $.16^{*}(.07)$ & $.11(.06)$ & $.11(.06)$ & $.10(.06)$ \\
\hline $\begin{array}{l}\text { Support for current } \\
\text { policies }\end{array}$ & & $.05(.04)$ & $.04(.04)$ & $.01(.04)$ & $-.01(.05)$ & $.02(.05)$ \\
\hline Cost of compliance & & $.03(.04)$ & $.03(.04)$ & $.03(.04)$ & $.03(.04)$ & .04 (.05) \\
\hline Negative emotions & & $.01(.04)$ & $.01(.04)$ & $.02(.04)$ & $.01(.04)$ & $-.02(.04)$ \\
\hline Impulsivity & & $.06(.07)$ & $.07(.07)$ & $.07(.06)$ & $.08(.07)$ & $.11(.07)$ \\
\hline Deterrence - Certainty & & & $.04(.04)$ & $.01(.04)$ & $-.01(.04)$ & $-.03(.04)$ \\
\hline Severity & & & $-.00(.03)$ & $.00(.03)$ & $.00(.03)$ & $-.02(.04)$ \\
\hline Social norms & & & $.00(.03)$ & $-.04(.03)$ & $-.04(.03)$ & $-.04(.03)$ \\
\hline Capacity to comply & & & & $.11 * *(.04)$ & $.11 * *(.04)$ & $.12 * *(.04)$ \\
\hline Clarity of measures & & & & $.05(.04)$ & $.04(.04)$ & $.06(.04)$ \\
\hline Knowledge & & & & $.09(.08)$ & $.10(.08)$ & $.08(.08)$ \\
\hline Opportunity to violate & & & & $-.08 *(.04)$ & $-.08 *(.04)$ & $-.07(.04)$ \\
\hline $\begin{array}{l}\text { Normative obligation to } \\
\text { obey the law }\end{array}$ & & & & & $.03(.03)$ & $.06 *(.03)$ \\
\hline Non-normative OOL & & & & & $.05(.04)$ & $.08(.04)$ \\
\hline Procedural justice & & & & & $.03(.07)$ & $.06(.07)$ \\
\hline Political orientation & & & & & & $-.21 * * *(.07)$ \\
\hline $\mathbf{R S q}$ & .02 & .05 & .05 & .08 & .09 & .14 \\
\hline$R S q$ (change) & .02 & .03 & .00 & .03 & .01 & .05 \\
\hline
\end{tabular}

Nb. * - Significant at the .05 level. ** - Significant at the .01 level. *** - Significant at the .001 level. Model $5-N=484$. 
Table 10.

Step-wise regression models of compliance "Lockdown" $(N=555)$

\begin{tabular}{|c|c|c|c|c|c|c|}
\hline & $\begin{array}{l}\text { Model } 0 . \\
\\
\text { Baseline } \\
\text { model } \\
\text { (controls } \\
\text { only) }\end{array}$ & $\begin{array}{c}\text { Model 1. } \\
+ \\
\text { Substantive } \\
\text { moral } \\
\text { support, } \\
\text { Costs of } \\
\text { compliance, } \\
\text { Impulsivity }\end{array}$ & $\begin{array}{l}+ \text { Deterrence, } \\
\text { Social Norms }\end{array}$ & $\begin{array}{l}\text { + Capacity, } \\
\text { opportunity }\end{array}$ & $\begin{array}{l}+ \text { Obligation to } \\
\text { obey the law }\end{array}$ & $\begin{array}{l}+ \text { Political } \\
\text { orientation }\end{array}$ \\
\hline & $\mathrm{b}(\mathrm{SE})$ & $\mathrm{b}(\mathrm{SE})$ & $\mathrm{b}(\mathrm{SE})$ & $\mathrm{b}(\mathrm{SE})$ & $\mathrm{b}(\mathrm{SE})$ & $\mathrm{b}(\mathrm{SE})$ \\
\hline Age & $-.00(.00)$ & $-.00(.00)$ & $-.00(.00)$ & $-.00(.00)$ & $-.00(.00)$ & $-.00(.00)$ \\
\hline \multicolumn{7}{|l|}{ Gender } \\
\hline i. female & $.34 * * *(.10)$ & $.35 * *(.11)$ & $.34 * *(.11)$ & $.28 *(.11)$ & $.23 *(.11)$ & $.26^{*}(.12)$ \\
\hline ii. non-binary & $.70 * * *(.14)$ & $.74 * * *(.16)$ & $.72 * * *(.16)$ & $.68 * * *(.18)$ & $.54 * *(.18)$ & $.69^{* * * *}(.18)$ \\
\hline SES pre-COVID-19 & $.03(.03)$ & $.01(.03)$ & $.02(.03)$ & $.01(.03)$ & $.01(.02)$ & $.02(.03)$ \\
\hline Health (self) & $.23 *(.11)$ & $.30 * *(.11)$ & $.30 * *(.11)$ & $.28 * *(.11)$ & $.27 * *(.11)$ & $.25 *(.11)$ \\
\hline Health (other) & $.03(.13)$ & $-.02(.13)$ & $-.02(.13)$ & $-.03(.12)$ & $-.03(.12)$ & $-.00(.14)$ \\
\hline Trust in media & $.05(.05)$ & $.03(.05)$ & $.03(.05)$ & $.02(.04)$ & $.02(.04)$ & $.01(.05)$ \\
\hline Perceived threat & & $-.08(.05)$ & $-.09(.05)$ & $-.08(.05)$ & $-.09(.05)$ & $-.12 *(.06)$ \\
\hline $\begin{array}{l}\text { Specific moral } \\
\text { alignment }\end{array}$ & & $.23 * * *(.07)$ & $.24 * * *(.07)$ & $.19 * *(.07)$ & $.18 * *(.07)$ & $.17 *(.07)$ \\
\hline $\begin{array}{l}\text { Support for current } \\
\text { policies }\end{array}$ & & $.00(.04)$ & $-.00(.04)$ & $-.02(.04)$ & $-.02(.04)$ & $-.01(.05)$ \\
\hline Cost of compliance & & $.07(.04)$ & $.07(.04)$ & $.07(.04)$ & $.07(.04)$ & $.06(.04)$ \\
\hline Negative emotions & & $-.09(.06)$ & $-.09(.06)$ & $-.08(.05)$ & $-.09(.05)$ & $-.10 *(.05)$ \\
\hline Impulsivity & & $-.04(.07)$ & $-.04(.07)$ & $-.03(.07)$ & $.00(.07)$ & $-.00(.08)$ \\
\hline Deterrence - Certainty & & & $.03(.04)$ & $.01(.04)$ & $-.01(.04)$ & $.01(.04)$ \\
\hline Severity & & & $-.02(.04)$ & $-.03(.04)$ & $-.02(.03)$ & $-.03(.04)$ \\
\hline Social norms & & & $-.02(.03)$ & $-.06(.03)$ & $-.06(.03)$ & $-.04(.03)$ \\
\hline Capacity to comply & & & & $.15^{* *}(.05)$ & $.16^{* * *}(.05)$ & $.19 * * *(.05)$ \\
\hline Clarity of measures & & & & $.02(.04)$ & $.02(.04)$ & $.04(.04)$ \\
\hline Knowledge & & & & $.20 * *(.08)$ & $.21 * *(.08)$ & $.24 *(.10)$ \\
\hline Opportunity to violate & & & & $-.01(.03)$ & $-.01(.03)$ & $-.01(.03)$ \\
\hline $\begin{array}{l}\text { Normative obligation to } \\
\text { obey the law }\end{array}$ & & & & & $.10 * * *(.03)$ & $.10 * *(.03)$ \\
\hline Non-normative OOL & & & & & $-.01(.04)$ & $-.01(.04)$ \\
\hline Procedural justice & & & & & $-.07(.05)$ & $-.05(.05)$ \\
\hline Political orientation & & & & & & $-.03(.06)$ \\
\hline RSq & .04 & .08 & .08 & .13 & .15 & .17 \\
\hline RSq (change) & .04 & .04 & .00 & .05 & .02 & .02 \\
\hline
\end{tabular}

Nb. $*$ - Significant at the .05 level. **-Significant at the .01 level. $* * *-$ Significant at the .001 level. Model $5-N=484$. 


\section{Discussion}

This study has looked at compliance with COVID-19 mitigation measures in the UK. At the time of data collection (April 6-8, 2020), the UK had been under lockdown for two weeks. This study finds that overall self-reported compliance in the UK is very high across the three measures; most people indicated that they are nearly always in compliance with the measures. Although one might argue that "nearly always" is not good enough when trying to contain a pandemic, ${ }^{22}$ we would like to stress that the data suggest a massive behavioural change has been achieved in just a few weeks. This is supported by GPS-data. Data from navigation app Citymapper, for instance, showed that the day after the lockdown, movement of Londoners had dropped to $15 \%$ compared to usual. ${ }^{23}$ Similarly, Google data tracking differences in human activity before and after the COVID-19 measures also showed large drops in retail and recreation $(75 \%)$, public transit $(71 \%)$, and visits to workplaces $(68 \%){ }^{24}$ Our data show a more detailed picture of the behavioural change due to the COVID-19 measures, as it shows us whether people comply with specific measures, namely keeping a safe distance, not meeting people outside their household, and staying at home apart from engaging in essential activities. Furthermore, this study provides data on the processes that have shaped compliance with the measures thus far. This allows us to better understand what actions authorities should take to maintain high levels of compliance during the course of the pandemic. We discuss several findings here.

First, it does not seem that people's overall substantive moral support for the measures played an important role in their compliance with the measures. For compliance with the social distancing measures (i.e., not meeting people and keeping a safe distance) we found no association with substantive moral support for the measures. Thus, whether people morally believed they should follow the measures, whether they perceived the disease as threatening, and whether they generally supported the measures and thought they were 
consistent, adequate, and proportional, did not influence their social distancing behaviour. We did find an association between compliance with the lockdown measure and two aspects of substantive moral support. We found a significant positive association with whether people morally believed that people should follow the measures. And, interestingly and counterintuitively, we found a negative association with whether people feared the disease (perceived threat). This means that, rather than staying in for their own health interests, people who have more fear of the virus show the opposite behaviour, and are more likely to break the rules and go out other than for essential activities. One possible explanation here may be that participants overall did not show great support for the measures (Table 3), which is in accordance with previous survey data in the UK that has shown that the public does not show great confidence in the government to manage COVID-19. ${ }^{25}$ This possibly explains the results of the lockdown measure, as people who perceive COVID-19 to be a great threat, but simultaneously do not support the government and their measures, may be inclined to disregard those same measures. Conversely, it could be the case that people that do not (or are not able to) stay at home, feel that they are more at risk of contracting COVID-19, and therefore fear the virus more. The precise nature of these relationships and interactions between variables should be examined more closely before conclusions can be made.

Furthermore, the data show that costs of compliance are not associated with compliance with the measures. People who had more negative emotions were less likely to comply, but only with the lockdown measures. This finding is in line with previous findings on strain and deviancy (Agnew 1992, 2006, Agnew et al. 2002, Agnew and White 1992, Baron 2004, Piquero and Sealock 2004, Botchkovar, Tittle, and Antonaccio 2009). At this point the data show that people do not report great costs of compliance or negative emotions (Table 3). Possibly, longer or recurring periods of lockdown or social distancing may cause 
increased costs of compliance and strain, which may then start to negatively affect compliance with all measures.

The data show no association between deterrence and compliance. This is in line with previous research findings that show that there is no conclusive evidence that stricter punishment alone can reduce offending (Nagin 2013, Nagin, Cullen, and Jonson 2009, Nagin and Pepper 2012, Simpson et al. 2014). Stricter punishment will only deter, if a minimum threshold level of certainty of punishment is met (Brown 1978, Chamlin 1991). It seems that our participants did not perceive high levels of punishment certainty (Table 3). This is not surprising, as it seems most of enforcement action is taken against the more flagrant forms of rule-breaking, such as house parties and public gatherings. ${ }^{26}$ Thus, if the authorities are unable to increase the certainty of punishment for less blatant forms of rule-breaking, our findings suggest that stricter sanctions (such as higher fines) are unlikely to have a positive effect on compliance. Moreover, the question is whether sufficient levels of certainty of punishment can be achieved for violating the COVID-19 mitigation measures in the UK, without strong surveillance measures such as for example mandatory smartphone tracking applications or facial recognition. While effective, such measures are controversial and spur debate about privacy. A recent study on this subject found that legitimacy and public trust in the police are crucial in this matter, as they influence acceptance of facial recognition technology and may diminish concerns about privacy (Bradford et al. 2020).

Furthermore, the data show that there is no association between descriptive social norms and compliance. Thus, UK residents' compliance is not influenced by seeing others comply with the rules. This is in contrast with previous research that has shown that social norms play an important role in compliance (Cialdini et al. 2006, Cialdini and Goldstein 2004, Goldstein, Cialdini, and Griskevicius 2008, Schultz et al. 2007), and recent research on 
COVID-19 mitigation measures in the US that also showed that greater perceived social norms positively affected compliance (Bogg and Milad 2020, Van Rooij et al. 2020) .

Most notably, the results show that compliance is dependent on people's own practical abilities. We did not find an association between clarity of measures and compliance, and correct knowledge of the current measures was only associated with compliance with lockdown measures. However, all three compliance measures were associated with capacity to comply, showing that people who are more practically able to follow the rules are more likely to do so. This indicates that authorities and employers must do everything they can to ensure that people can work from home, maintain social distance, and stay indoors apart from essential activities. When this is not possible, authorities and employers should enable people to maintain a safe distance while traveling to work, for example, and avoid overloaded public transport. ${ }^{27}$

Opportunity to violate was only associated with the measure that requires people to not meet others outside of their household. Thus, for keeping a safe distance and lockdown measures, the opportunity to violate the measures did not cause more people to actually do so. This shows that, in the UK, increasing people's abilities to comply with the rules is more important for compliance than reducing the opportunities to violate implemented measures. Additionally, as it is specifically the "not meeting other people" measure that is influenced by opportunity, current practices of closing down non-essential businesses or public venues ${ }^{28}$ may indeed be helpful to increase compliance, as this reduces the opportunity to meet others there.

For obligation to obey the law, the results are mixed. The data show that neither nonnormative obligation (i.e., compliance out of fear of the authorities themselves) nor procedural justice are associated with compliance. However, a greater normative obligation to obey the law was associated with all compliance measures, in line with previous research 
on this topic (Fine et al. 2020, Fine, Van Rooij, et al. 2016, Tyler 2017, Tyler 1997, 2006, Nagin and Telep 2017, Walters and Bolger 2019). This shows that, in the UK, it is not fear for authorities or belief in the authorities' fairness that shapes compliance, but rather people's intrinsic belief that they should obey the law.

For political orientation, we found only an association with the measure that tells people to keep a safe distance. The data show that people with more progressive political beliefs are more likely to comply with the distancing measure, but not the other compliance measures. This is (partly) in line with an earlier study that found that, in the US, partisanship was the most important predictor for health behaviour and attitudes in the COVID-19 pandemic (Kushner Gadarian, Wallace Goodman, and Pepinsky 2020). However, overall, political orientation does not seem to play a big role in compliance with COVID-19 measures in the UK, similar to our previous findings in the US (Van Rooij et al. 2020). Another recent study, with a sample that largely consisted of British citizens, also did not find that political ideology was associated with compliance with COVID-19 measures. They concluded that, in crisis, people may "forgo their ideological commitments and behave consistently with governmental advice in pursuit of a common public health good" (Harper et al. 2020, 15). Another possible explanation is that the COVID-19 measures may have brought about a shift in people's attitudes toward politics, as campaign promises that the British government made before election can no longer be held. Where at first strong promises were made for investments to boost the economy in the neglected northern/middle territories of the UK, the current crisis may cause the inequality between these areas and the richer south most likely to become even larger, ${ }^{29}$ especially since now these resources have to be used as a safety net for the economic consequences of the lockdown. Furthermore, we did not ask for our participants' vote in Brexit, which does seem to continue to shape opinion beyond partisanship. $^{30}$ 


\section{Conclusion}

The present study analysed what made UK residents comply with the COVID-19 mitigation measures. The most important findings were that compliance depended on people's capacity to comply with the rules, and the normative obligation they feel to obey the law. As such, compliance was not associated with deterrence or obedience out of fear, but the intrinsic motivation and capacity people have to comply with the measures.

If we compare these results with the US data (Van Rooij et al. 2020), we see that there are some important similarities. Namely, health-related fears and deterrence do not predict compliance on any of the measures, but practical capacity to comply, and to a lesser extent opportunity to violate, do.

However, some variables that were important in the US sample, such as social norms and impulsivity, did not predict compliance in the UK. An important difference between the two samples was the sampling method. For the UK, we used a stratified sampling method resulting in a representative sample that was more evenly divided across age. Most notably, the average age was much higher (46.22 vs. 34.46 in the US), and the proportion of people younger than 30 was $20 \%$ (vs. $50 \%$ in the US). As younger people may have different interests or motivations for (non-)compliance (Oosterhoff and Palmer 2020), this may partially explain the differences between the studies.

Another major difference is that in the UK normative obligation to obey the law (OOL) predicted compliance where in the US it did not. Here, there may be broader sociopolitical differences at play with how Americans and UK citizens see themselves in relation to the legal system and governmental authorities. Unfortunately our survey did not have measures that allow us to analyse this further.

This study has some important limitations. First, we have limited ourselves to preliminary analyses, and have not yet conducted interactions or models of sets of variables 
to understand how different variables may mediate others. Furthermore, we did not measure objective behaviour, but used self-report measures for compliance, which may result in underestimations of rule-breaking behaviour as people want to give socially desirable answers. However, existing research shows that self-reported behaviour can give a good image of objective compliance measures when surveys are utilized, as was the case in the present study (Garber et al. 2004, Bachmann et al. 1999, Dieltjens et al. 2013, Rauscher et al. 1993, Ridgers et al. 2012). Recently, scholars have also examined whether self-report measures can be used to track objective compliance with the COVID-19 mitigation measures. A study in the US found that self-report measures of social distancing reflect actual behaviour at the individual and group level (Gollwitzer et al. 2020). Moreover, a Danish study found no evidence for social desirability bias in survey results of compliance with COVID-19 mitigation measures (Larsen, Nyrup, and Petersen 2020).

The present study has several practical implications that may aid authorities in the UK to sustain compliance with COVID-19 mitigation measures. The main focus of interventions and public messaging should not be on scaring citizens into compliance, either through emphasizing risks to health or risks of punishment. Instead, three things matter in compliance studied in this survey. First, it is important to ensure the legitimacy of the measures and authorities that can foster people's sense of obligation to obey (Tyler 2017, Tyler 2006) and to appeal to their intrinsic motivation to obey the measures (Posch et al. 2020). Second, authorities should practically enable people to comply with the measures (e.g., by making sure they can work from home or avoid crowded public transport). Third, authorities should reduce practical opportunities for proximity with non-household members.

These findings provide a warning for easing the current restrictions too soon. UK authorities should consider very carefully what the behavioural consequences might be of their plans to ease the current measures. Once people are allowed to go out more and engage 
in work and social activities, maintaining social distancing will become much harder. Our data show that when people have the opportunity for unsafe proximity, they will violate social distancing measures. And should this happen and more people be found in violation of safe distancing measures, authorities must refrain from unfair law enforcement that undermines people's sense of obligation to obey the rules (Walters and Bolger 2019), and hence may erode a vital source of compliance this study found in the UK. Moving past the first peak of the pandemic, maintaining compliance will thus likely become more challenging, and simplistic strategies that focus on harder punishment will not be sufficient to ensure that people keep a safe distance. 


\section{Literature}

Adler, Nancy E, Elissa S Epel, Grace Castellazzo, and Jeannette R Ickovics. 2000. "Relationship of subjective and objective social status with psychological and physiological functioning: Preliminary data in healthy white women." Health Psychology 19 (6):586.

Agnew, Robert. 1992. "Foundation for a general strain theory of crime and delinquency." Criminology 30 (1):47-88.

Agnew, Robert. 2006. "Pressured into crime: An overview of general strain theory."

Agnew, Robert, Timothy Brezina, John Paul Wright, and Francis T Cullen. 2002. "Strain, personality traits, and delinquency: Extending general strain theory." Criminology 40 (1):43-72.

Agnew, Robert, and Helene Raskin White. 1992. "An empirical test of general strain theory." Criminology 30 (4):475-500.

Ansolabehere, Stephen, and Brian F Schaffner. 2014. "Does survey mode still matter? Findings from a 2010 multi-mode comparison." Political Analysis 22 (3):285-303.

Apel, Robert. 2013. "Sanctions, perceptions, and crime: Implications for criminal deterrence." Journal of quantitative criminology 29 (1):67-101.

Bachmann, Laura H, Joan Stephens, Charity M Richey, and EDWARD W HOOK III. 1999. "Measured versus self-reported compliance with doxycycline therapy for chlamydiaassociated syndromes: high therapeutic success rates despite poor compliance." Sexually transmitted diseases 26 (5):272-278.

Baker, Thomas, and Jacinta M Gau. 2018. "Female offenders' perceptions of police procedural justice and their obligation to obey the law." Crime \& Delinquency 64 (6):758-781.

Baron, Stephen W. 2004. "General strain, street youth and crime: A test of Agnew's revised theory." Criminology 42 (2):457-484.

Becker, Gary S. 1968. "Crime and Punishment, An Economic Approach." Journal of Political Economy 76:169-217.

Bogg, T., and E. Milad. 2020. "[Pre-print]. Slowing the Spread of COVID-19: Dempgraphic, personality an social cognition predictors of guideline adherence in a representative U.S. sample.". doi: 10.31234/osf.io/yc2gq.

Botchkovar, Ekaterina V, Charles R Tittle, and Olena Antonaccio. 2009. "General strain theory: Additional evidence using cross - cultural data." Criminology 47 (1):131-176.

Bradford, Ben, Julia Yesberg, Jonathan Jackson, and Paul Dawson. 2020. "Live Facial Recognition: Trust and Legitimacy as Predictors of Public Support for Police Use of New Technology." British Journal of Criminology.

Brown, Don W. 1978. "Arrest rates and crime rates: when does a tipping effect occur?" Social Forces 57 (2):671-682.

Chamlin, Mitchell B. 1991. "A longitudinal analysis of the arrest-crime relationship: A further examination of the tipping effect." Justice Quarterly 8 (2):187-199.

Cialdini, Robert B, Linda J Demaine, Brad J Sagarin, Daniel W Barrett, Kelton Rhoads, and Patricia L Winter. 2006. "Managing social norms for persuasive impact." Social Influence 1 (1):3-15.

Cialdini, Robert B., and Noah J. Goldstein. 2004. "Social influence: compliance and conformity." Annual Review of Psychology 55:591-621. doi: 10.1146/annurev.psych.55.090902.142015.

Clarke, Ronald VG. 1980. "" Situational" Crime Prevention: Theory and Practice." The British Journal of Criminology 20 (2):136-147. 
Cohen, Lawrence E, and Marcus Felson. 1979. "Social change and crime rate trends: A routine activity approach." American sociological review 44 (4):588-608.

Couper, Mick P. 2017. "New developments in survey data collection." Annual Review of Sociology 43:121-145.

Couper, Mick P, Garret Gremel, William Axinn, Heidi Guyer, James Wagner, and Brady T West. 2018. "New options for national population surveys: The implications of internet and smartphone coverage." Social Science Research 73:221-235.

Dieltjens, Marijke, Marc J Braem, Anneclaire VMT Vroegop, Kristien Wouters, Johan A Verbraecken, Wilfried A De Backer, Paul H Van de Heyning, and Olivier M Vanderveken. 2013. "Objectively measured vs self-reported compliance during oral appliance therapy for sleep-disordered breathing." Chest 144 (5):1495-1502.

Donovan, Jenny L, and David R Blake. 1992. "Patient non-compliance: deviance or reasoned decision-making?" Social science \& medicine 34 (5):507-513.

Estévez, Estefanía, and Nicholas P Emler. 2010. "A structural modelling approach to predict adolescent offending behaviour from family, school and community factors." European Journal on Criminal Policy and Research 16 (4):207-220.

Everett, JAC, C Colombatto, V Chituc, WJ Brady, and MJ Crockett. 2020. "[Pre-print]. The effectiveness of moral messages on public health behavioral intentions during the COVID-19 pandemic." doi: 10.31234/osf.io/9yqs8.

Feldman, Yuval. 2018. The law of good people: Challenging states' ability to regulate human behavior: Cambridge University Press.

Fetzer, T, M Witte, L Hensel, J Jachimowicz, J Haushofer, A Ivchenko, S Caria, E Reutskaja, C Roth, S Fiorin, M Gomez, G Kraft-Todd, F Goetz, and E Yoeli. 2020. "[Pre-print]. Global behaviors and preceptions in the COVID-19 pandemic." doi: 10.31234/osf.io/3kfmh.

Fine, Adam D, Zachary Rowan, and Cortney Simmons. 2019. "Do politics Trump race in determining America's youths' perceptions of law enforcement?" Journal of Criminal Justice 61:48-57.

Fine, Adam, Laurence Steinberg, Paul J Frick, and Elizabeth Cauffman. 2016. "Self-control assessments and implications for predicting adolescent offending." Journal of youth and adolescence 45 (4):701-712.

Fine, Adam, April Thomas, Benjamin van Rooij, and Elizabeth Cauffman. 2020. "AgeGraded Differences and Parental Influences on Adolescents' Obligation to Obey the Law." Journal of Developmental and Life-Course Criminology:1-18.

Fine, Adam, Benjamin Van Rooij, Yuval Feldman, Shaul Shalvi, Margerita Leib, Eline Scheper, and Elizabeth Cauffman. 2016. "Rule Orientation and Behavior:

Development and Validation of a Scale Measuring Individual Acceptance of Rule Violation." Psychology, Public Policy, and Law 22 (3):314-329.

Friedman, Lawrence M. 2016. Impact: Harvard University Press.

Garber, Mathew C, David P Nau, Steven R Erickson, James E Aikens, and Joseph B Lawrence. 2004. "The concordance of self-report with other measures of medication adherence: a summary of the literature." Medical care:649-652.

Gau, Jacinta M. 2014. "Procedural justice and police legitimacy: A test of measurement and structure." American Journal of Criminal Justice 39 (2):187-205.

Goldstein, Noah J., Robert B. Cialdini, and Vladas Griskevicius. 2008. "A room with a viewpoint: using social norms to motivate environmental conservation in hotels." Journal of Consumer Research 35:472-482.

Gollwitzer, A, C Martel, Marshall. K, JM Höhs, and JA Bargh. 2020. "[Pre-print]. Connecting Self-Reported Social Distancing to Real-World Behavior at the Individual and U.S. State Level." doi: 10.31234/osf.io/kvnwp. 
Gottfredson, Michael R, and Travis Hirschi. 1990. A general theory of crime: Stanford University Press.

Harper, CA, LP Satchell, D Fido, and RD Latzman. 2020. "[Pre-print]. Functional fear predicts public health compliance in the COVID-19 pandemic." doi: 10.31234/osf.io/jkfu3.

Hasson, Yossi, Maya Tamir, Kea S Brahms, J Christopher Cohrs, and Eran Halperin. 2018. "Are liberals and conservatives equally motivated to feel empathy toward others?" Personality and Social Psychology Bulletin 44 (10):1449-1459.

Hines, Denise A, Emily M Douglas, and Sehar Mahmood. 2010. "The effects of survey administration on disclosure rates to sensitive items among men: A comparison of an internet panel sample with a RDD telephone sample." Computers in human behavior 26 (6):1327-1335.

I\&O Research, and Universiteit Twente. 2020. Rapport: Het Corona- en het eenzaamheidsvirus. Amsterdam: I\&O Research.

Kushner Gadarian, S, S Wallace Goodman, and TB Pepinsky. 2020. "[Pre-print]. Partisanship, health behavior, and policy attitudes in the early stages of the COVID19 pandemic." doi: 10.2139/ssrn.3562796

Larsen, M.V., J. Nyrup, and M. B. Petersen. 2020. "[Pre-print]. Do survey estimates of the public's compliance with COVID-19 regulation suffer from social desirability bias?". doi: 10.31234/osf.io/cy4hk.

Lunn, PD, S Timmons, CA Belton, M Barjaková, H Julienne, and C Lavin. 2020. "[Preprint]. Motivating social distancing during the Covid-19 pandemic: An online experiment." doi: 10.31234/osf.io/x4agb.

McCright, Aaron M, Katherine Dentzman, Meghan Charters, and Thomas Dietz. 2013. "The influence of political ideology on trust in science." Environmental Research Letters 8 (4):044029.

Nagin, Daniel S. 2013. "Deterrence in the Twenty-first Century." Crime and Justice 42 (1):199-263.

Nagin, Daniel S, Francis T Cullen, and Cheryl Lero Jonson. 2009. "Imprisonment and reoffending." Crime and justice 38 (1):115-200.

Nagin, Daniel S, and John V Pepper, eds. 2012. Deterrence and the death penalty. Washington DC: National Academies Press.

Nagin, Daniel S, and Cody W Telep. 2017. "Procedural justice and legal compliance." Annual Review of Law and Social Science 13:5-28.

Oosterhoff, B, and CA Palmer. 2020. "[Pre-print]. Psychological correlates of news monitoring, social distancing, disinfecting, and hoarding behaviors among US adolescents during the COVID-19 pandemic." doi: 10.31234/osf.io/rpcy4.

Paternoster, Raymond, and Sally Simpson. 1993. "A rational choice theory of corporate crime." Routine activity and rational choice 5:37.

Piquero, Nicole Leeper, and Miriam D Sealock. 2004. "Gender and general strain theory: A preliminary test of Broidy and Agnew's gender/GST hypotheses." Justice quarterly 21 (1):125-158.

Plohl, N., and B. Musil. 2020. "[Pre-print]. Modeling compliance with COVID-19 prevention guidelines: The critical role of trust in science." doi: 10.31234/osf.io/6a2cx.

Posch, Krisztian, Jonathan Jackson, Ben Bradford, and Sarah Macqueen. 2020. "' Truly Free Consent"? Clarifying the Nature of Police Legitimacy using Causal Mediation Analysis." Journal of Experimental Criminology.

Pratt, Travis C, and K Lloyd. 2021 (forthcoming). "Self-control and offending." In The Cambridge Handbook on Compliance, edited by B Van Rooij and D Sokol. Cambridge, UK: Cambridge University Press. 
Prior, Markus. 2013. "Media and political polarization." Annual Review of Political Science 16:101-127.

Rauscher, Helmuth, Dieter Formanek, Wolfgang Popp, and Hartmut Zwick. 1993. "Selfreported vs measured compliance with nasal CPAP for obstructive sleep apnea." Chest 103 (6):1675-1680.

Reisig, Michael D, Jason Bratton, and Marc G Gertz. 2007. "The construct validity and refinement of process-based policing measures." Criminal justice and behavior 34 (8):1005-1028.

Ridgers, Nicola D, Anna Timperio, David Crawford, and Jo Salmon. 2012. "Validity of a brief self-report instrument for assessing compliance with physical activity guidelines amongst adolescents." Journal of Science and Medicine in Sport 15 (2):136-141.

Schultz, P. Wesley, Jessica M. Nolan, Robert B. Cialdini, Noah J. Goldstein, and Vladas Griskevicius. 2007. "The constructive, destructive, and reconstructive power of social norms." Psychological Science 18 (5):429-434.

Simpson, Sally, Melissa Rorie, Mariel Elise Alper, Natalie Schell-Busey, William Laufer, and N Craig Smith. 2014. "Corporate Crime Deterrence: A Systematic Review." Campbell Systematic Reviews 10 (4):5-88.

Spohr, Dominic. 2017. "Fake news and ideological polarization: Filter bubbles and selective exposure on social media." Business Information Review 34 (3):150-160.

Tankebe, Justice, Michael D Reisig, and Xia Wang. 2016. "A multidimensional model of police legitimacy: A cross-cultural assessment." Law and Human Behavior 40 (1):11.

Tyler, Tom. 2017. "Procedural justice and policing: A rush to judgment?" Annual Review of Law and Social Science 13:29-53.

Tyler, Tom R. 1997. "Procedural Fairness and Compliance with the Law." Swiss Journal of Economics and Statistics 133 (2):p. 219-240.

Tyler, Tom R. 2006. Why People Obey the Law. Princeton: Princeton University Press.

Van Rooij, Benjamin. 2021 (Forthcoming). "Do people know the law? Empirical evidence about legal knowledge and its implications for compliance." In Cambridge Handbook of Compliance, edited by Benjamin van Rooij and D Daniel Sokol. Cambridge, UK: Cambridge University Press.

Van Rooij, Benjamin, Anne Leonore de Bruijn, Christopher P. Reinders Folmer, Emmeke Kooistra, Malouke Esra Kuiper, Megan Brownlee, Elke Olthuis, and Adam Fine. 2020. "Compliance with COVID-19 Mitigation Measures in the United States " Working Paper posted on PsyArXiv psyarxiv.com/qymu3.

Van Rooij, Benjamin, and D Daniel Sokol. 2021 (Forthcoming). "Compliance as the interaction between rules and behavior (Introduction to Cambridge Handbook of Compliance)." In Cambridge Handbook of Compliance, edited by D Daniel Sokol and Benjamin Van Rooij. Cambridge, UK: Cambridge University Press.

Walker, Patrick, Charles Whittaker, Oliver Watson, Marc Baguelin, Kylie Ainslie, Sangeeta Bhatia, Samir Bhatt, Adhiratha Boonyasiri, Olivia Boyd, Lorenzo Cattarino, Zulma Cucunubá, Gina Cuomo-Dannenburg, Amy Dighe, Christl Donnelly, Ilaria Dorigatti, Sabine van Elsland, Rich FitzJohn, Seth Flaxman, Han Fu, Katy Gaythorpe, Lily Geidelberg, Nicholas Grassly, Will Green, Arran Hamlet, Katharina Hauck, David Haw, Sarah Hayes, Wes Hinsley, Natsuko Imai, David Jorgensen, Edward Knock, Daniel Laydon, Swapnil Mishra, Gemma Nedjati-Gilani, Lucy Okell, Steven Riley, Hayley Thompson, Juliette Unwin, Robert Verity, Michaela Vollmer, Caroline Walters, Hao Wei Wang, Yuanrong Wang, Peter Winskill, Xiaoyue Xi, Neil Ferguson, and Azra Ghan. 2020. "The Global Impact of COVID-19 and Strategies for Mitigation and Suppression." Imperial College Covid-19 Reports (12). 
Walters, Glenn D, and P Colin Bolger. 2019. "Procedural justice perceptions, legitimacy beliefs, and compliance with the law: A meta-analysis." Journal of experimental Criminology 15 (3):341-372.

Weinberger, Daniel A, and Gary E Schwartz. 1990. "Distress and restraint as superordinate dimensions of self - reported adjustment: A typological perspective." Journal of Personality 58 (2):381-417.

Wojcik, Sean P, Arpine Hovasapian, Jesse Graham, Matt Motyl, and Peter H Ditto. 2015. "Conservatives report, but liberals display, greater happiness." Science 347 (6227):1243-1246.

Wolfe, Scott E, Justin Nix, Robert Kaminski, and Jeff Rojek. 2016. "Is the effect of procedural justice on police legitimacy invariant? Testing the generality of procedural justice and competing antecedents of legitimacy." Journal of quantitative criminology 32 (2):253-282.

Zhang, XiaoChi, Lars Kuchinke, Marcella L Woud, Julia Velten, and Jürgen Margraf. 2017. "Survey method matters: Online/offline questionnaires and face-to-face or telephone interviews differ." Computers in Human Behavior 71:172-180. 


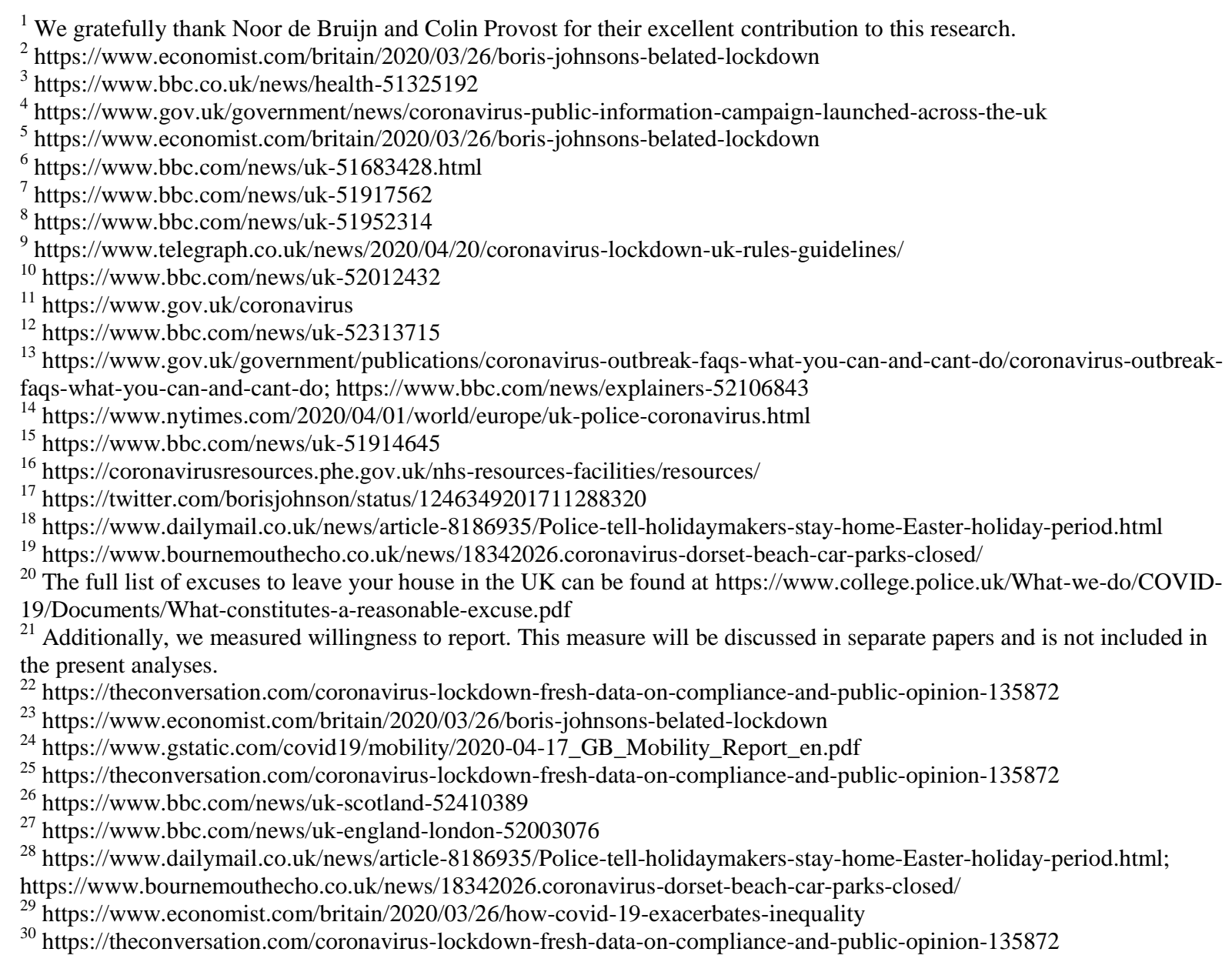

